\title{
Inclusões Dinâmicas em Escalas Temporais: Existência de Soluções sob a Hipótese de Semicontinuidade Inferior
}

I.L.D. SANTOS ${ }^{1}$, G.N. SILVA ${ }^{2}$ Departamento de Matemática Aplicada, IBILCE, UNESP - Univ Estadual Paulista, 15054-000 São José do Rio Preto, SP, Brasil.

L. BARBANTI ${ }^{3}$ Departamento de Matemática, FEIS, UNESP - Univ Estadual Paulista, 15385-000 Ilha Solteira, SP, Brasil.

Resumo. Neste trabalho consideramos o problema de inclusão diferencial em escalas temporais cujo campo vetorial é uma multifunção, ou seja, uma função que mapea pontos a conjuntos. O trabalho fornece condições de existência sem exigir compacidade do campo vetorial; exige apenas que ele seja convexo, fechado e semicontínuo inferior. Em trabalhos anteriores na literatura, ou o campo é escalar ou exige-se que este, além de convexo, seja compacto e tenha o gráfico fechado.

Palavras-chave. inclusões dinâmicas, escalas temporais, existência de soluções

\section{Introdução}

Inclusões dinâmicas com campos vetoriais em escalas temporais tem sido pouco exploradas na literatura. Entretanto, quando o campo é escalar tem recebido bastante atenção. Ver por exemplo os trabalhos [3], [4], [7], [10] e [12]. No trabalho [21] é tratado o caso vetorial, mas sob as hipóteses de que o campo é convexo, compacto e com gráfico fechado.

Utilizando o método de soluções superiores e inferiores, em [4] é provado a existência de soluções para inclusões dinâmicas de primeira ordem em escalas temporais com condições de fronteira gerais. Já em [12] prova-se a existência de soluções para inclusões dinâmicas de primeira ordem em escalas temporais com condições iniciais não-locais.

Resultados de existência de soluções para inclusões dinâmicas de segunda ordem em escalas temporais com condições de fronteira usando o método de soluções superiores e inferiores podem ser encontrados em [3] e [10].

Neste trabalho provamos a existência de soluções para o problema de inclusão dinâmica vetorial de primeira ordem sob as hipóteses de o campo vetorial associado

\footnotetext{
${ }^{1}$ iguerluis@yahoo.com.br; O autor agradece ao apoio financeiro da CAPES na forma de bolsa de doutorado

${ }^{2}$ gsilva@ibilce.unesp.br; Este autor agradece ao apoio financeiro recebido da FAPESP - Proc: 2009/18643-0 e a bolsa de Produtividade em Pesquisa concedida pelo CNPq

${ }^{3}$ barbanti@mat.feis.unesp.br
} 
com a inclusão ser convexo, fechado e semicontínuo inferior. O resultado generaliza resultados de existência para o caso contínuo (ver por exemplo, [5]) e, de certa forma, resultados em escalas temporais em que o campo é escalar. O trabalho também generaliza, para inclusões dinâmicas, o resultado de existência (Teorema $4.3,[14])$, provado para equações dinâmicas em escalas temporais.

Este trabalho é organizado da seguinte forma. Na seção 2 revisamos resultados e conceitos básicos sobre cálculo diferenical, medida e integral em escalas de tempo. Na seção 3 enunciamos e provamos o novo resultado de existência de soluções para o problema de inclusões dinâmicas em escalas de tempo. Na seção 4 fazemos comentários finais em relação a contribuição deste trabalho.

\section{Cálculo, Medida e Integração em Escala Tempo- ral}

Nesta seção revisamos os resultados e conceitos básicos sobre a análise em escalas de tempo. Relebramos os conceitos de derivadas de funções escalares e vetoriais em escalas de tempo. A seguir introduzimos a medida $\Delta$ e a integral Delta de Lebesgue em escalas de tempo, bem como algumas propriedades necessárias ao desenvolvimento da existência de soluções quando o campo é semicontínuo inferior.

O cálculo em escalas temporais foi introduzido por Hilger [16] como uma forma de unificar o cálculo diferencial e de diferenças. Entretanto, o estudo sobre escalas ramificou-se e hoje existe uma vasta literatura sobre o assunto, das quais citamos [1], [2], [3], [13], [18].

\subsection{Cálculo em escala temporal}

Neste trabalho $\mathbb{T}$ é uma escala temporal, isto é, um subconjunto fechado do $\mathbb{R}^{n}$. Definimos a função $\sigma: \mathbb{T} \rightarrow \mathbb{T}$ por $\sigma(t)=\inf \{s \in \mathbb{T}: s>t\}$ e a função $\rho: \mathbb{T} \rightarrow \mathbb{T}$ como sendo $\rho(t)=\sup \{s \in \mathbb{T}: s<t\}$. Estamos supondo que inf $\emptyset=\sup \mathbb{T}$ e $\sup \emptyset=\inf \mathbb{T}$. Definimos a função $\mu: \mathbb{T} \rightarrow[0,+\infty)$ da seguinte forma $\mu(t)=\sigma(t)-t$.

Lema 2.1 ([11]). Se $\mathbb{T}$ é uma escala temporal então existem $I \subset \mathbb{N} e\left\{t_{i}\right\}_{i \in I} \subset \mathbb{T}$ tal que

$$
R S:=\{t \in \mathbb{T}: t<\sigma(t)\}=\left\{t_{i}\right\}_{i \in I} .
$$

Se $A \subset \mathbb{R}$, definimos o conjunto $A_{\mathbb{T}}$ como $A_{\mathbb{T}}=A \cap \mathbb{T}$. Se sup $\mathbb{T}<+\infty$ definimos

$$
\mathbb{T}^{\kappa}=\mathbb{T} \backslash(\rho(\sup \mathbb{T}), \sup \mathbb{T}]_{\mathbb{T}}
$$

e se $\sup \mathbb{T}=+\infty$ definimos $\mathbb{T}^{\kappa}=\mathbb{T}$.

Considere uma função $f: \mathbb{T} \rightarrow \mathbb{R}$ e $t \in \mathbb{T}^{\kappa}$. Se $\xi \in \mathbb{R}$ é tal que, para todo $\varepsilon>0$ existe $\delta>0$ de modo que

$$
|f(\sigma(t))-f(s)-\xi(\sigma(t)-s)| \leq \varepsilon|\sigma(t)-s|
$$

para todo $s \in(t-\delta, t+\delta)_{\mathbb{T}}$, dizemos que $\xi$ é a delta derivada de $f$ em $t$ e denotamos $\xi=f^{\Delta}(t)$. 
Considere uma escala temporal $\mathbb{T}$, uma função $f: \mathbb{T} \rightarrow \mathbb{R}^{n}$ e $t \in \mathbb{T}^{\kappa}$. Dizemos que $f$ é $\Delta$-diferenciável em $t$ se cada função coordenada $f_{i}: \mathbb{T} \rightarrow \mathbb{R}$ for $\Delta$-diferenciável em $t$. Neste caso $f^{\Delta}(t)=\left(f_{1}^{\Delta}(t), \ldots, f_{n}^{\Delta}(t)\right)$.

Teorema 2.1 ([9]). Considere uma escala temporal $\mathbb{T}$, uma função $f: \mathbb{T} \rightarrow \mathbb{R}^{n} e$ $t \in \mathbb{T}^{\kappa}$. Valem as seguintes propriedades:

(i) Se $f$ é $\Delta$-diferenciável em $t$ então $f$ é continua em $t$.

(ii) Se $f$ é contínua em $t$ e $\sigma(t)>t$, então $f$ é $\Delta$-diferenciável em $t$. Além disso,

$$
f^{\Delta}(t)=\frac{f(\sigma(t))-f(t)}{\mu(t)} .
$$

(iii) Se $\sigma(t)=t$, então $f$ é $\Delta$-diferenciável em $t$ se, e somente se, o limite

$$
\lim _{s \rightarrow \underset{\mathbb{T}}{\rightarrow} t} \frac{f(t)-f(s)}{t-s}
$$

existe como um elemento de $\mathbb{R}^{n}$. Neste caso

$$
f^{\Delta}(t)=\lim _{s \underset{\mathbb{T}}{\rightarrow} t} \frac{f(t)-f(s)}{t-s} .
$$

(iv) Se fé $\Delta$-diferenciável em $t$, então $f(\sigma(t))=f(t)+\mu(t) f^{\Delta}(t)$.

Teorema 2.2 ([9]). Seja $\mathbb{T}$ uma escala temporal. Suponha que as funções $f, g$ : $\mathbb{T} \rightarrow \mathbb{R}$ são $\Delta$-diferenciáveis em $t \in \mathbb{T}^{\kappa}$. Então:

(i) A soma $f+g: \mathbb{T} \rightarrow \mathbb{R}$ é $\Delta$-diferenciável em $t$ e vale a relação

$$
(f+g)^{\Delta}(t)=f^{\Delta}(t)+g^{\Delta}(t) .
$$

(ii) O produto f.g: $\mathbb{T} \rightarrow \mathbb{R}$ é $\Delta$-diferenciável em $t$. Além disso,

$$
\begin{aligned}
(f . g)^{\Delta}(t) & =f^{\Delta}(t) g(t)+f(\sigma(t)) g^{\Delta}(t) \\
& =f(t) g^{\Delta}(t)+f^{\Delta}(t) g(\sigma(t)) .
\end{aligned}
$$

Corolário 2.2.1 ([9]). Seja $\mathbb{T}$ uma escala temporal. Suponha que as funções $f, g$ : $\mathbb{T} \rightarrow \mathbb{R}^{n}$ são $\Delta$-diferenciáveis em $t \in \mathbb{T}^{\kappa}$. Então a soma $f+g: \mathbb{T} \rightarrow \mathbb{R}^{n}$ é $\Delta$ diferenciável em t. Além disso,

$$
(f+g)^{\Delta}(t)=f^{\Delta}(t)+g^{\Delta}(t) .
$$

Definição 2.1 ([17]). Considere um conjunto $E \subset C\left[\mathbb{T}, \mathbb{R}^{m}\right]$, em que $C\left[\mathbb{T}, \mathbb{R}^{m}\right]$ é o conjunto $f: \mathbb{T} \rightarrow \mathbb{R}^{m}$. Dizemos que E é equicontínuo em $t \in \mathbb{T}$ se para todo $\varepsilon>0$, existe $\delta>0$, tal que

$$
\|f(s)-f(t)\|<\varepsilon
$$

quando $s \in(t-\delta, t+\delta) \cap \mathbb{T}$ e $f \in E$.

Um conjunto $E \subset C\left[\mathbb{T}, \mathbb{R}^{m}\right]$ é equicontínuo se for equicontínuo em cada $t \in \mathbb{T}$.

Lema 2.2 ([17]). Considere uma escala temporal $\mathbb{T}$ compacta e um conjunto $E \subset$ $C\left[\mathbb{T}, \mathbb{R}^{m}\right]$ limitado na norma do máximo. Se E é equicontínuo então toda sequência de $E$ possui subsequência que converge uniformemente em $\mathbb{T}$. 


\subsection{Medida exterior}

Denote por $\mathfrak{F}$ a coleção de subintervalos de $\mathbb{T}$ da forma $[\tilde{a}, \tilde{b})_{\mathbb{T}}=\{t \in \mathbb{T}: \tilde{a} \leq$ $t<\tilde{b}\}$, sendo $\tilde{a}, \tilde{b} \in \mathbb{T}$. O intervalo $[\tilde{a}, \tilde{a})_{\mathbb{T}}$ é entendido como o conjunto vazio.

Seja $E \subset \mathbb{T}$ um subconjunto qualquer. Se existe pelo menos uma sequência de intervalos $\left[a_{j}, b_{j}\right)_{\mathbb{T}} \in \mathfrak{F}$ tal que $E \subset \bigcup_{j}\left[a_{j}, b_{j}\right)_{\mathbb{T}}$, definimos a medida exterior de $E$ como

$$
m^{*}(E)=\inf \left\{\sum_{k=1}^{+\infty}\left(b_{k}-a_{k}\right): E \subset \bigcup_{k}\left[a_{k}, b_{k}\right)_{\mathbb{T}},\left[a_{k}, b_{k}\right)_{\mathbb{T}} \in \mathfrak{F}\right\} .
$$

Se não existir uma tal cobertura de $E$ definimos $m^{*}(E)=+\infty$.

Convencionamos que $m^{*}(\emptyset)=0$ e denotamos a medida exterior em $\mathbb{R}$ por $\lambda^{*}$.

Lema $2.3([15])$. Se $c, d \in \mathbb{T}$ e $c<d$, então $m^{*}\left([c, d)_{\mathbb{T}}\right)=d-c$.

Lema 2.4 ([11]). Se $E \subset[a, b)_{\mathbb{T}}$, então

$$
m^{*}(E)=\sum_{i \in I_{E}}\left(\sigma\left(t_{i}\right)-t_{i}\right)+\lambda^{*}(E) .
$$

É consequência imediata deste Lema o seguinte corolário.

Corolário 2.2.2. Seja $E \subset[a, b)_{\mathbb{T}}$. Se $m^{*}(E)=\lambda^{*}(E)$ então $E \cap R S=\emptyset$. Reciprocamente, se $E \cap R S=\emptyset$, isto é, $E \subset\{t \in \mathbb{T}: \sigma(t)=t\}$, então $m^{*}(E)=\lambda^{*}(E)$.

\subsection{Conjuntos mensuráveis}

Um conjunto $E \subset \mathbb{T}$ é chamado de $\Delta$-mensurável (Lebesgue $\Delta$-mensurável) se

$$
m^{*}(A)=m^{*}(A \cap E)+m^{*}(A \cap(\mathbb{T} \backslash E))
$$

para cada conjunto $A \subset \mathbb{T}$.

Proposição 2.1 ([11]). Seja $E \subset \mathbb{T}$. Então $E$ é $\Delta$-mensurável se, e somente se, E é Lebesgue mensurável.

Teorema 2.3 ([8],[19]). A família $\Delta$ de conjuntos $\Delta$-mensuráveis é uma $\sigma$-álgebra.

O Teorema 2.3 permite-nos definir a medida $m^{*}: \Delta \rightarrow[0,+\infty]$ que chamamos de $\Delta$-medida de Lebesgue e denotamos por $m^{*} \equiv \mu_{\Delta}$.

Dizemos que uma proposição $P$ vale $\Delta$-quase sempre $(\Delta$-a.e.) em $\mathbb{T} \backslash\{b\}$, se o conjunto $N$ dado por $N=\{t \in \mathbb{T} \backslash\{b\}: P$ nao vale em $t\}$ é tal que $\mu_{\Delta}(N)=0$.

Se $M=\{t \in \mathbb{T} \backslash\{b\}: P$ vale em $t\}$ segue que $N=[a, b)_{\mathbb{T}} \backslash M$. Como $M=[a, b)_{\mathbb{T}} \backslash N$ e $N \in \Delta$ concluímos que $M \in \Delta$.

Lema 2.5. Se duas proposições $P$ e $Q$ valem $\Delta$-quase sempre em $\mathbb{T} \backslash\{b\}$, então $P \cap Q$ vale $\Delta$-quase sempre em $\mathbb{T} \backslash\{b\}$.

Demonstração. Temos que

$$
N=\{t \in \mathbb{T} \backslash\{b\}: P \cap Q \text { nao vale em } t\} \subset
$$

$$
N_{1}=\{t \in \mathbb{T} \backslash\{b\}: P \text { nao vale em } t\} \bigcup N_{2}=\{t \in \mathbb{T} \backslash\{b\}: Q \text { nao vale em } t\}
$$

e então $\mu_{\Delta}(N) \leq \mu_{\Delta}\left(N_{1} \cup N_{2}\right) \leq \mu_{\Delta}\left(N_{1}\right)+\mu_{\Delta}\left(N_{2}\right)=0$. 


\section{4. $\Delta$-Integral de Lebesgue}

Os livros clássicos [6] e [20] contêm as propriedades básicas de funções mensuráveis e funções integráveis no caso em que as medidas são abstratas ou de Lebesgue. Aqui especializamos os conceitos e resultados para a $\sigma$-álgebra $\Delta$ e a integral correspondente, sem repetir as demonstrações que podem ser facilmente obtidas nos livros citados. Assim, quando um resultado estiver sem demonstração e sem citação é porque ele pode ser enconctrado em [6] ou [20].

Definição 2.2. Dizemos que uma função $f: \mathbb{T} \rightarrow[-\infty,+\infty]$ é $\Delta$-mensurável se para cada $\alpha \in \mathbb{R}$ o conjunto $\{t \in \mathbb{T}: f(t)<\alpha\}$ é $\Delta$-mensurável.

Definição 2.3. Dizemos que uma função $f: \mathbb{T} \rightarrow \mathbb{R}^{n}$ é $\Delta$-mensurável se cada função coordenada $f_{i}: \mathbb{T} \rightarrow \mathbb{R}$ é $\Delta$-mensurável.

Definição 2.4. Se $E \in \Delta$, denotamos por $L_{1}(E)$ o conjunto das funções $f: \mathbb{T} \rightarrow \mathbb{R}$ $\Delta$-mensuráveis e integráveis em $E$.

Definição 2.5. Seja $f: \mathbb{T} \rightarrow \mathbb{R}^{n}$ uma função $\Delta$-mensurável e $E \in \Delta$. Dizemos que $f$ é integrável em $E$ se cada função coordenada $f_{i}: \mathbb{T} \rightarrow \mathbb{R}$ é integrável em $E$.

Definição 2.6. Se $E \in \Delta$, denotamos por $L_{1}\left(E, \mathbb{R}^{n}\right)$ o conjunto das funções $f: \mathbb{T} \rightarrow \mathbb{R}^{n} \Delta$-mensuráveis e integráveis em $E$.

Como consequência do Teorema da convergência dominada de Lebesgue ([6] ou [20]) temos o seguinte corolário.

Corolário 2.3.3. Considere uma sequência $f_{n}: \mathbb{T} \rightarrow \mathbb{R}$ de funções $\Delta$-mensuráveis. Seja $\phi: \mathbb{T} \rightarrow[0,+\infty)$ uma função em $L_{1}\left([a, b)_{\mathbb{T}}\right)$ tal que para cada $n$ temos $\left|f_{n}(t)\right| \leq$ $\phi(t)$ para todo $t \in \mathbb{T}$.

Se $A \subset[a, b)_{\mathbb{T}}$ é $\Delta$-mensurável então

$$
\int_{A} \liminf f_{n}(s) \Delta s \leq \liminf \int_{A} f_{n}(s) \Delta s .
$$

Demonstração. A demonstração segue do Teorema da convergência dominada de Lebesgue [6, 20] e será omitida.

Lema 2.6. Se $E \in \Delta$ e $f \in L_{1}\left(E, \mathbb{R}^{n}\right)$ então $\|f\| \in L_{1}(E)$.

Demonstração. Para cada $t \in \mathbb{T}$ temos que

$$
\|f(t)\|=\left\|\left(f_{1}(t), \ldots, f_{n}(t)\right)\right\| \leq\left|f_{1}(t)\right|+\ldots+\left|f_{n}(t)\right| .
$$

Como $\left|f_{1}\right|, \ldots,\left|f_{n}\right| \in L_{1}(E)$ segue que $\|f\| \in L_{1}(E)$.

Definição 2.7. Dada uma função $f: \mathbb{T} \rightarrow \mathbb{R}^{n}$ definimos $\tilde{f}:[a, b] \rightarrow \mathbb{R}^{n}$ como

$$
\tilde{f}(t)=\left\{\begin{array}{l}
f(t), \quad t \in \mathbb{T} \\
f\left(t_{i}\right), \quad t \in\left(t_{i}, \sigma\left(t_{i}\right)\right) \text { para algum } i \in I
\end{array}\right.
$$

sendo $I \subset \mathbb{N} e\left\{t_{i}\right\}_{i \in I} \subset \mathbb{T}$ tal que

$$
R S:=\{t \in \mathbb{T}: t<\sigma(t)\}=\left\{t_{i}\right\}_{i \in I} .
$$


Proposição 2.2 ([11]). Considere uma função $f: \mathbb{T} \rightarrow \mathbb{R}$. Então $f$ é $\Delta$-mensurável se, e somente se, $f$ é $\mathcal{L}$-mensurável.

Corolário 2.3.4. Seja $f: \mathbb{T} \rightarrow \mathbb{R}^{n}$ uma função dada. Então $f$ é $\Delta$-mensurável se, e somente se, $\tilde{f}$ é $\mathcal{L}$-mensurável.

Definição 2.8. Se $E \subset \mathbb{T}$ defina

$$
\tilde{E}=E \cup \bigcup_{i \in I_{E}}\left(t_{i}, \sigma\left(t_{i}\right)\right) .
$$

Teorema 2.4 ([11]). Seja um conjunto $\Delta$-mensurável $E \subset \mathbb{T}$ tal que $b \notin E$. Seja $f: \mathbb{T} \rightarrow \mathbb{R}$ uma função $\Delta$-mensurável e $\tilde{f}:[a, b] \rightarrow \mathbb{R}$ a extensão de $f$ dada pela Definição 2.7.

Então, $f \in L_{1}(E)$ se, e somente se, $\tilde{f} \in L_{1}(\tilde{E})$. Neste caso,

$$
\int_{E} f(s) \Delta s=\int_{\tilde{E}} \tilde{f}(s) d s .
$$

Corolário 2.4.5. Seja $E \subset \mathbb{T}$ um conjunto $\Delta$-mensurável tal que $b \notin E$. Seja $f: \mathbb{T} \rightarrow \mathbb{R}^{n}$ uma função $\Delta$-mensurável e $\tilde{f}:[a, b] \rightarrow \mathbb{R}^{n}$ a extensão de $f$ dada pela Definição 2.7.

Então, $f \in L_{1}\left(E, \mathbb{R}^{n}\right)$ se, e somente se, $\tilde{f} \in L_{1}\left(\tilde{E}, \mathbb{R}^{n}\right)$. Neste caso,

$$
\int_{E} f(s) \Delta s=\int_{\tilde{E}} \tilde{f}(s) d s .
$$

Proposição 2.3. Considere uma função $g \in L_{1}\left([a, b)_{\mathbb{T}}\right)$. Suponha que

$$
\int_{[c, d)_{\mathbb{T}}} g(s) \Delta s=0
$$

para cada $c, d \in \mathbb{T}$ tal que $c<d$. Então $g(t)=0 \Delta$-a.e. $t \in[a, b)_{\mathbb{T}}$.

Demonstração. Observe inicialmente que $\tilde{g} \in L_{1}([a, b))$, já que $g \in L_{1}\left([a, b)_{\mathbb{T}}\right)$.

Seja $t \in[a, b)_{\mathbb{T}}$ fixado arbitrariamente. Se $\sigma(t)>t$, segue que

$$
0=\int_{[t, \sigma(t))_{\mathrm{T}}} g(s) \Delta s=\int_{\{t\}} g(s) \Delta s=g(t)\{\sigma(t)-t\}
$$

e então $g(t)=0$.

Se $\sigma(t)=t$ e $t$ é um ponto de Lebesgue de $\tilde{g}$, seja $\left\{\delta_{i}\right\}$ uma sequência tal que $\delta_{i} \downarrow 0$ e $t+\delta_{i} \in[a, b)_{\mathbb{T}}$ para cada $i$. Do Teorema 2.4 e de [20] temos

$$
0=\lim \frac{1}{\delta_{i}} \int_{\left[t, t+\delta_{i}\right)_{\mathbb{T}}} g(s) \Delta s=\lim \frac{1}{\delta_{i}} \int_{\left[t, t+\delta_{i}\right)} \tilde{g}(s) d s=\tilde{g}(t)=g(t)
$$

já que $t \in \mathbb{T}$.

Assim, se $D=\left\{s \in[a, b)_{\mathbb{T}}: g(s) \neq 0\right\}$ segue que $D \subset A \cap B$, onde

$$
A=\left\{s \in[a, b)_{\mathbb{T}}: \sigma(s)=s\right\}
$$


e $B$ o conjunto dos pontos $s \in[a, b)_{\mathbb{T}}$ tal que $s$ não é ponto de Lebesgue de $\tilde{g}$. Do Lema 2.2 .2 e de [20] segue que

$$
m^{*}(D) \leq m^{*}(A \cap B)=\lambda^{*}(A \cap B) \leq \lambda^{*}(B)=0
$$

e portanto $g(t)=0 \Delta$-a.e. $t \in[a, b)_{\mathbb{T}}$.

Proposição 2.4. Seja $g \in L_{1}\left([a, b)_{\mathbb{T}}\right)$ e suponha que

$$
\int_{[c, d)_{\mathbb{T}}} g(s) \Delta s \geq 0
$$

para cada $c, d \in \mathbb{T}$ tal que $c<d$. Então $g(t) \geq 0 \Delta$-a.e. $t \in[a, b)_{\mathbb{T}}$.

Demonstração. Considere $t \in[a, b)_{\mathbb{T}}$ fixado arbitrariamente. Se $\sigma(t)>t$, segue que

$$
0 \leq \int_{[t, \sigma(t))_{\mathbb{T}}} g(s) \Delta s=\int_{\{t\}} g(s) \Delta s=g(t)\{\sigma(t)-t\}
$$

e então $g(t) \geq 0$.

Se $\sigma(t)=t$ e $t$ é um ponto de Lebesgue de $\tilde{g}$, considere uma sequência $\left\{\delta_{i}\right\}$ tal que $\delta_{i} \downarrow 0$ e $t+\delta_{i} \in[a, b)_{\mathbb{T}}$ para cada $i$. Do Teorema 2.4 e de [20] temos que

$$
0 \leq \lim \frac{1}{\delta_{i}} \int_{\left[t, t+\delta_{i}\right)_{\mathbb{T}}} g(s) \Delta s=\lim \frac{1}{\delta_{i}} \int_{\left[t, t+\delta_{i}\right)} \tilde{g}(s) d s=\tilde{g}(t)=g(t)
$$

já que $t \in \mathbb{T}$.

Assim, se $D=\left\{s \in[a, b)_{\mathbb{T}}: g(s)<0\right\}$ temos que $D \subset A \cap B$, sendo

$$
A=\left\{s \in[a, b)_{\mathbb{T}}: \sigma(s)=s\right\}
$$

e $B$ o conjunto dos pontos $s \in[a, b)_{\mathbb{T}}$ tal que $s$ não é ponto de Lebesgue de $\tilde{g}$. Do Lema 2.2.2 e de [20] segue que

$$
m^{*}(D) \leq m^{*}(A \cap B)=\lambda^{*}(A \cap B) \leq \lambda^{*}(B)=0
$$

e portanto $g(t) \geq 0 \Delta$-a.e. $t \in[a, b)_{\mathbb{T}}$.

Proposição 2.5. Considere uma função $g \in L_{1}\left([a, b)_{\mathbb{T}}, \mathbb{R}^{n}\right)$. Suponha que

$$
\int_{[c, d)_{\mathbb{T}}} g(s) \Delta s=0
$$

para cada $c, d \in \mathbb{T}$ tal que $c<d$. Então $g(t)=0 \Delta$-a.e. $t \in[a, b)_{\mathbb{T}}$.

Demonstração. Temos que $g=\left(g_{1}, \ldots, g_{n}\right)$, sendo cada função coordenada $g_{i} \in$ $L_{1}\left([a, b)_{\mathbb{T}}\right)$. Da Proposição 2.3 temos $g_{i}(t)=0 \quad \Delta-$ a.e. $t \in[a, b)_{\mathbb{T}}$ para cada $i \in\{1, \ldots, n\}$. Assim,

$$
N=\left\{s \in[a, b)_{\mathbb{T}}: g(s) \neq 0\right\} \subset \bigcup_{i=1}^{n} N_{i}
$$

onde $N_{i}=\left\{s \in[a, b)_{\mathbb{T}}: g_{i}(s) \neq 0\right\} . \log \mathrm{O}$

$$
m^{*}(N) \leq m^{*}\left(\cup N_{i}\right) \leq \sum_{i} m^{*}\left(N_{i}\right)=0
$$

e podemos concluir que $g(t)=0 \quad \Delta-$ a.e. $t \in[a, b)_{\mathbb{T}}$. 


\section{Existência de Soluções para o Problema de In- clusão Dinâmica}

Na Subseção 3.1 recordamos alguns conceitos e propriedades de multifunções, os quais são utilizados na demonstração do resultado da Subseção 3.2. Introduzimos o problema de inclusão dinâmica em escalas de tempo, enunciamos e provamos o resultado principal sobre a existência de soluções na Subseção 3.2.

\subsection{Multifunções}

Definição 3.1. Sejam $M$ e $N$ espaços métricos e considere uma multifunção $F$ : $M \rightsquigarrow N$, isto é, uma função que leva pontos de $M$ a subconjuntos do espaço $N$. Diz-se que $F$ é semicontinua inferior em $x_{0} \in M$ se para cada $y_{0} \in F\left(x_{0}\right)$ e para toda vizinhança $V\left(y_{0}\right)$ de $y_{0}$ existe uma vizinhança $V\left(x_{0}\right)$ de $x_{0}$ tal que

$$
F(x) \cap V\left(y_{0}\right) \neq \emptyset
$$

para todo $x \in V\left(x_{0}\right)$.

Definição 3.2. Dada uma multifunção $F: M \rightsquigarrow N$, dizemos que uma função $f: M \rightarrow N$ é uma seleção de $F$ quando $f(x) \in F(x)$ para todo $x \in M$.

Teorema 3.1 ([5]). Considere um espaço métrico $M$, um espaço de Banach $N$ e uma multifunção $F: M \rightsquigarrow N$ semicontínua inferior. Se $F$ é não-vazia, convexa $e$ fechada então F admite uma seleção contínua.

\subsection{Resultado principal}

Teorema 3.2. Seja uma multifunção $F: \mathbb{T} \times \mathbb{R}^{n} \rightsquigarrow \mathbb{R}^{n}$ não-vazia, convexa $e$ fechada. Se $F$ é semicontinua inferior, então existe $b_{1} \in \mathbb{T} \backslash\{a\}$ tal que o problema de valor inicial

$$
\left\{\begin{array}{l}
x^{\Delta}(t) \in F(t, x(t)) \quad \Delta-\text { a.e. } t \in\left[a, b_{1}\right)_{\mathbb{T}} \\
x(a)=x_{0}
\end{array}\right.
$$

possui solução.

Demonstração. Do Teorema 3.1 existe uma seleção contínua $f: \mathbb{T} \times \mathbb{R}^{n} \rightarrow \mathbb{R}^{n}$ de $F$.

Se $\sigma(a)>a$ tome $b_{1}=\sigma(a)$. Defina o arco $x:\left[a, b_{1}\right]_{\mathbb{T}} \rightarrow \mathbb{R}^{n} \operatorname{como} x(a)=x_{0} \mathrm{e}$ $x\left(b_{1}\right)=\mu(a) f(a, x(a))+x(a)$. Logo

$$
x^{\Delta}(a)=\frac{x(\sigma(a))-x(a)}{\mu(a)}=f(a, x(a))
$$

e então

$$
x^{\Delta}(t)=f(t, x(t)) \in F(t, x(t))
$$


quando $t \in\left[a, b_{1}\right)_{\mathbb{T}}=a$.

Suponha que $\sigma(a)=a$. Fixado um número $L>0$, como $f$ é contínua existe $M>1$ tal que

$$
\|f(u, w)\| \leq \frac{M}{n}
$$

quando $u \in \mathbb{T}$ e $\left\|w-x_{0}\right\| \leq L$. Seja $b_{1} \in \mathbb{T}$ tal que $b_{1}>a$ e $\left(b_{1}-a\right) M \leq L$.

Considere o seguinte conjunto convexo

$K:=\left\{x \in C\left(\left[a, b_{1}\right]_{\mathbb{T}}, \mathbb{R}^{n}\right): x(a)=x_{0}, x\right.$ de Lipschitz com constante $\left.M\right\}$.

Se $x \in K$, para cada $t \in\left[a, b_{1}\right]_{\mathbb{T}}$ temos

$$
\|x(t)\| \leq M|t-a|+\left\|x_{0}\right\| \leq M\left(b_{1}-a\right)+\left\|x_{0}\right\| .
$$

Como $K$ é equicontínuo segue do Lema 2.2 que o conjunto $K$ é compacto.

Defina a aplicação $T: K \rightarrow C\left(\left[a, b_{1}\right]_{\mathbb{T}}, \mathbb{R}^{n}\right)$ como

$$
(T(x))(t)=x_{0}+\int_{[a, t)_{\mathbb{T}}} f(\tau, x(\tau)) \Delta \tau
$$

para cada $t \in\left[a, b_{1}\right]_{\mathbb{T}}$ e $x \in K$.

Se $x \in K$ para todo $\tau \in\left[a, b_{1}\right]_{\mathbb{T}}$ temos

$$
\left\|x(\tau)-x_{0}\right\| \leq M|\tau-a| \leq M\left(b_{1}-a\right) \leq L
$$

e então $\|f(\tau, x(\tau))\| \leq \frac{M}{n}$. Assim, se $s, t \in\left[a, b_{1}\right]_{\mathbb{T}}$ e $s>t$ segue que

$$
\begin{aligned}
\|(T(x))(s)-(T(x))(t)\| & =\left\|\int_{[a, s)_{\mathbb{T}}} f(\tau, x(\tau)) \Delta \tau-\int_{[a, t)_{\mathbb{T}}} f(\tau, x(\tau)) \Delta \tau\right\| \\
& =\left\|\int_{[t, s)_{\mathbb{T}}} f(\tau, x(\tau)) \Delta \tau\right\| \\
& \leq M(s-t)
\end{aligned}
$$

A desigualdade (3.2) é uma consequência de (3.1).

De modo análogo, se $s, t \in\left[a, b_{1}\right]_{\mathbb{T}}$ e $s<t$ temos que

$$
\|(T(x))(s)-(T(x))(t)\| \leq M(t-s)
$$

e portanto $T(x) \in K$.

Se $D:=\left\{w \in \mathbb{R}^{n}:\left\|w-x_{0}\right\| \leq L\right\}$ segue que $f$ é uniformemente contínua em $\mathbb{T} \times D$. Assim, dado $\varepsilon>0$ existe $\delta>0$ tal que

$$
\|f(u)-f(v)\|<\frac{\varepsilon}{n\left(b_{1}-a\right)}
$$

quando $u, v \in \mathbb{T} \times D$ e $\|u-v\|<\delta$.

Tome $\bar{x} \in K$ arbitrariamente. Se $x \in K$ satisfaz

$$
\|x-\bar{x}\|_{\infty}<\delta
$$


para cada $s \in\left[a, b_{1}\right]_{\mathbb{T}}$ temos

$$
\begin{aligned}
\|(T(x))(s)-(T(\bar{x}))(s)\| & =\left\|x_{0}+\int_{[a, s)_{\mathbb{T}}} f(\tau, x(\tau)) \Delta \tau-x_{0}-\int_{[a, s)_{\mathbb{T}}} f(\tau, \bar{x}(\tau)) \Delta \tau\right\| \\
& \leq n \int_{[a, s)_{\mathbb{T}}}\|f(\tau, x(\tau))-f(\tau, \bar{x}(\tau))\| \Delta \tau \\
& \leq n \int_{[a, s)_{\mathbb{T}}} \frac{\varepsilon}{n\left(b_{1}-a\right)} \Delta \tau \quad \text { (esta desigualdade vem de (3.3)) } \\
& \leq n \int_{\left[a, b_{1}\right)_{\mathbb{T}}} \frac{\varepsilon}{n\left(b_{1}-a\right)} \Delta \tau=\varepsilon .
\end{aligned}
$$

Então $\|T(x)-T(\bar{x})\|_{\infty} \leq \varepsilon$ e portanto $T$ é contínua em $\bar{x}$.

Do Teorema do Ponto Fixo de Schauder [5] existe $x_{*} \in K$ tal que $T\left(x_{*}\right)=x_{*}$.

Se $x \in K$ defina o arco $z:\left[a, b_{1}\right]_{\mathbb{T}} \rightarrow \mathbb{R}^{n}$ por

$$
z(s)=x_{0}+\int_{[a, s)_{\mathbb{T}}} f(\tau, x(\tau)) \Delta \tau
$$

para cada $s \in\left[a, b_{1}\right]_{\mathbb{T}}$.

Para todo $t \in\left[a, b_{1}\right]_{\mathbb{T}}$ temos

$$
z(t)=z(a)+\int_{[a, t)_{\mathbb{T}}} z^{\Delta}(\tau) \Delta \tau .
$$

Como $z(a)=x_{0}$, para cada $t \in\left[a, b_{1}\right]_{\mathbb{T}}$ segue que

$$
\int_{[a, t)_{\mathbb{T}}} z^{\Delta}(\tau) \Delta \tau=\int_{[a, t)_{\mathbb{T}}} f(\tau, x(\tau)) \Delta \tau .
$$

Assim, se para cada $s \in\left[a, b_{1}\right]_{\mathbb{T}}$ a função $g \in L_{1}\left(\left[a, b_{1}\right)_{\mathbb{T}}, \mathbb{R}^{n}\right)$ é definida como

$$
g(s)=z^{\Delta}(s)-f(s, x(s))
$$

temos que

$$
\int_{[a, t)_{\mathbb{T}}} g(\tau) \Delta \tau=0
$$

para todo $t \in\left[a, b_{1}\right]_{\mathbb{T}}$.

Seja $c, d \in\left[a, b_{1}\right]_{\mathbb{T}}$ tal que $c<d$. Logo

$$
\int_{[c, d)_{\mathrm{T}}} g(\tau) \Delta \tau=\int_{[a, d)_{\mathrm{T}}} g(\tau) \Delta \tau-\int_{[a, c)_{\mathrm{T}}} g(\tau) \Delta \tau=0
$$

e da Proposição 2.5 concluímos que

$$
g(t)=z^{\Delta}(t)-f(t, x(t))=0 \quad \Delta-\text { a.e. } t \in\left[a, b_{1}\right)_{\mathbb{T}}
$$

isto é,

$$
z^{\Delta}(t)=f(t, x(t)) \quad \Delta-\text { a.e. } t \in\left[a, b_{1}\right)_{\mathbb{T}} .
$$

Portanto

$$
\left\{\begin{array}{l}
x_{*}^{\Delta}(t)=f\left(t, x_{*}(t)\right) \in F\left(t, x_{*}(t)\right) \quad \Delta-\text { a.e. } t \in\left[a, b_{1}\right)_{\mathbb{T}} \\
x_{*}(a)=x_{0} .
\end{array}\right.
$$




\title{
4. Considerações Finais
}

No Teorema 3.2 provamos a existência de solução para uma inclusão dinâmica usando uma seleção contínua do campo vetorial desta inclusão dinâmica. A solução obtida é uma solução usual da equação dinâmica, cujo campo vetorial é uma seleção contínua.

Por outro lado, quando o campo vetorial da inclusão dinâmica é uma multifunção semicontínua superior, [21] prova a existência de solução usando uma seleção arbitrária de seu campo vetorial. Para isso supõe-se que o campo vetorial seja uma multifunção não-vazia, convexa, compacta e satisfaça uma condição de crescimento linear. Nesse caso, a solução será uma solução de Euler da equação dinâmica, cujo campo vetorial é uma tal seleção.

Finalmente, gostariamos de agradecer aos revisores anônimos que muito contribuiram para a melhoria técnica e redação final deste trabalho.

\begin{abstract}
In this paper we consider the problem of differential inclusion in time scales whose vector field is a multifunction, that is, a function that maps points to sets. It is provided conditions of existence without requiring compactness of the vector field; it is required that the vector field is closed, convex, and lower semicontinuous. In previous work in literature, it is required that the field is either scalar or compact, convex, and has closed graph.
\end{abstract}

Keywords. Dynamic inclusions, time scales, existence of solutions.

\section{Referências}

[1] M. Adivar, Y.N. Raffoul, Existence of resolvent for Volterra integral equations on time scales, Bulletin of the Australian Mathematical Society, 82, No.1 (2010), 139-155.

[2] E. Akin-Bohner, Y.N. Raffoul, C.C. Tisdell, Exponenctial stability in functional dynamic equations on time scales, Communications in Mathematical Analysis, 9, No.1 (2010), 93-108.

[3] E. Akin-Bohner, S. Sun, Existence of solutions for second-order dynamic inclusions, Int. J. Dynamical Systems and Differential Equations, 3, No.1-2 (2011), 24-37.

[4] F.M. Atici, D.C. Biles, First order dynamic inclusions on time scales, Journal of Mathematical Analysis and Applications, 292, No.1 (2004), 222-237.

[5] J.P. Aubin, A. Cellina, "Differential Inclusions", Springer-Verlag, Berlin, 1984.

[6] R.G. Bartle, "The Elements of Integration and Lebesgue Measure", John Wiley and Sons, New York, 1995.

[7] A. Belarbi, M. Benchohra, A. Ouahab, Existence results for impulsive dynamic inclusions on time scales, Electronic Journal of Qualitative Theory of Differential Equations, No. 12 (2005), 22. 
[8] M. Bohner, A. Peterson, "Advances in Dynamic Equations on Time Scales, Birkhauser", Boston, 2003.

[9] M. Bohner, A. Peterson, "Dynamic Equations on Time Scales", Birkhauser, Boston, 2001.

[10] M. Bohner, C.C. Tisdell, Second order dynamic inclusions, Journal of Nonlinear Mathematical Physics, 12, No.2 (2005), 36-45.

[11] A. Cabada, D.R. Vivero, Expression of the Lebesgue $\Delta$-integral on time scales as a usual Lebesgue integral; application to the calculus of $\Delta$-antiderivatives, Mathematical and Computer Modelling, 43, No.1-2 (2006), 194-207.

[12] Y.K. Chang, W.T. Li, Existence results for dynamic inclusions on time scales with nonlocal initial conditions, Computers and Mathematics with Applications, 53, No. 1 (2007), 12-20.

[13] L. Erbe, T.S. Hassan, A. Peterson, Oscillation of third order nonlinear functional dynamic equations on time scales, Differential Equations and Dynamical Systems, 18, No.1-2 (2010), 199-227.

[14] H. Gilbert, Existence theorems for first-order equations on time scales with $\Delta$-Caratheódory functions, Advances in Difference Equations, 2010, (2010),120 .

[15] G.S. Guseinov, Integration on time scales, Journal of Mathematical Analysis and Applications, 285, No.1 (2003), 107-127.

[16] S. Hilger, Analysis on measure chains- a unified approach to continuous and discrete calculus, Results in Mathematics, 18, No.1-2 (1990), 18-56.

[17] E.L. Lima, "Espaços Métricos", Coleção Projeto Euclides, Rio de Janeiro, 2003.

[18] A. Liu, M. Bohner, Gronwall-OuIang-type integral inequalities on time scales, Journal of Inequalities and Applications, 10, (2010), 15.

[19] H.L. Royden, "Real Analysis", Collier-Macmillan Limited, London, 1968.

[20] W. Rudin, "Real and Complex Analysis", third edition, McGraw-Hill Book Company, New York, 1987.

[21] I.L.D. Santos, G.N. Silva, Absolute continuity and existence of solutions to vector dynamic inclusions in time scales, "Technical Report", Department of Computing and Statistics, IBILCE, UNESP - Univ Estadual Paulista, São José do Rio Preto, SP, 2011. 